\title{
Correction to: Importance of evaluating protein glycosylation in pluripotent stem cell-derived cardiomyocytes for research and clinical applications
}

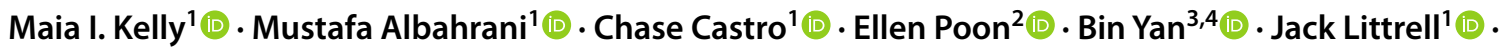 \\ Matthew Waas ${ }^{1,5}$ (D) Kenneth R. Boheler ${ }^{6}$ (D) Rebekah L. Gundry ${ }^{1}$
}

Published online: 1 June 2021

(c) Springer-Verlag GmbH Germany, part of Springer Nature 2021

\section{Correction to: Pflügers Archiv - European Journal of Physiology (2021)}

$$
\text { https://doi.org/10.1007/s00424-021-02554-x }
$$

The original article was published with an error. The reference citation [17] including the Reference should be deleted from the list.

The original article has been corrected.

Publisher's Note Springer Nature remains neutral with regard to jurisdictional claims in published maps and institutional affiliations.

The original article can be found online at https://doi.org/10.1007/ s00424-021-02554-x.

\section{Rebekah L. Gundry}

1 CardiOmics Program, Center for Heart and Vascular, Research, Division of Cardiovascular Medicine, Department of Cellular and Integrative Physiology, University of Nebraska Medical Center, Omaha, NE 68198, USA

2 Department of Medicine and Therapeutics, Centre for Cardiovascular Genomics and Medicine, Hong Kong Hub of Pediatric Excellence, Lui Che Woo Institute of Innovative Medicine, The Chinese University of Hong Kong, Hong Kong, HKSAR, China

3 Department of Computer Sciences, The University of Hong Kong, Hong Kong, HKSAR, China

4 The Second Afliated Hospital and Yuying Children's Hospital of Wenzhou Medical University, Wenzhou, Zhejiang, China

5 Present Address: Princess Margaret Cancer Centre, University Health Network, Toronto, ON M5G 0A3, Canada

6 Whiting School of Engineering, Department of Biomedical Engineering, Traylor Building, The Johns Hopkins, University, Baltimore, MD 21205, USA 